\title{
Charge Generation and Recombination in Diketopyrrolopyrrole Polymer: Fullerene Bulk Heterojunctions Studied by Transient Absorption and Time-Resolved Microwave Conductivity
}

Ryuzi Katoh, ${ }^{1,2^{\star}}$ Hiroyuki Matsuzaki, ${ }^{2}$ Akihiro Furube, ${ }^{2,3}$ Prashant Sonar, ${ }^{4,}$ Evan L. Williams, ${ }^{5}$ Chellappan Vijila, ${ }^{5}$ Gomathy Sandhya Subramanian, ${ }^{5}$ Sergey Gorelik, ${ }^{5}$ and Jonathan Hobley $^{6}$

${ }^{1}$ Department of Chemical Biology and Applied Chemistry, College of Engineering, Nihon University, Koriyama, Fukushima 963-8642, Japan

${ }^{2}$ National Institute of Advanced Industrial Science and Technology (AIST), Tsukuba Central 2, 1-1-1 Umezono, Tsukuba, Ibaraki 305-8568, Japan

${ }^{3}$ Department of Optical Science, Tokushima University, 2-1, Minamijosanjima-cho, Tokushima, 770-8506, Japan

${ }^{4}$ School of Chemistry, Physics and Mechanical Engineering, Queensland University of Technology (QUT), GPO Box 2434, Brisbane QLD 4001, Australia

${ }^{5}$ Institute of Materials Research and Engineering (IMRE), Agency for Science, Technology and Research (A*STAR), 2 Fusionopolis Way, Innovis, \#08-03, Singapore 138634

${ }^{6}$ Faculty of Science, Universiti Brunei Darussalam, Jalan Tungku Link, BE1410, Negara, Brunei Darussalam

${ }^{*}$ Corresponding author

E-mail: rkatoh@chem.ce.nihon-u.ac.jp

\begin{abstract}
Charge generation and recombination dynamics in organic photovoltaic bulk heterojunction films comprising the donor polymer, PDPP-TNT (Poly \{3,6-dithiophene2-yl-2,5-di(2-octyldodecyl)- pyrrolo[3,4-c]pyrrole-1,4-dione-alt-naphthalene\}), blended with the fullerene acceptor, $\mathrm{PC}_{71} \mathrm{BM}$ ([6,6]-phenyl $\mathrm{C}_{71}$-butyric acid methyl ester), have been studied. The charge carrier generation process was studied using femtosecond transient absorption and it was found that the efficiency of charge generation is not dominated by geminate recombination but rather is limited by exciton diffusion in the films. Highly sensitive nanosecond transient absorption (ns-TA) and time-resolved microwave conductivity (TRMC) were used to study charge recombination. From ns-TA measurements, we obtained a recombination rate constant of $1 \times 10^{-9} \mathrm{~cm}^{3} \mathrm{~s}^{-1}$ and found that charge recombination is limited by the diffusion of charge carriers (Langevin-type recombination). TRMC signals were
\end{abstract}


comparable with ns-TA on shorter time scales. However, in contrast to ns-TA, the TRMC signal contained an additional long-lived component. The fast decay at shorter time scales is attributed to the recombination of the majority of the charge carriers. The long-lived component is assigned to a small population of charge carriers with high mobility, suggesting they are located in isolated, crystalline domains within the bulk heterojunction. These findings are discussed in relation to the morphology of the blend film, fluorescence quenching properties, and device performance including photoinduced charge extraction by linearly increasing voltage (PhotoCELIV) measurements described in our previous publications.

\section{Introduction}

Organic photovoltaics (OPV) based on conjugated polymers have attracted much attention for their light weight, low cost, mechanical flexibility and potential for production of large-scale devices. ${ }^{1,2}$ Much effort has been made to improve power conversion efficiency (PCE). Recently, 11.5\% PCE has been achieved. ${ }^{3}$ The bulk hetero-junction (BHJ) structure is commonly employed in such OPV devices. It consists of an interpenetrating network of conjugated polymer (donor) and fullerene derivative (acceptor). Although various materials have been examined, a mixture of poly(3-hexylthiophene) and [6,6]phenyl $\mathrm{C}_{61}$-butyric acid methyl ester ( $\mathrm{P} 3 \mathrm{HT}$ and $\mathrm{PC}_{61} \mathrm{BM}$, respectively) has been widely used as a prototype material. The principles of photo-energy conversion are considered as follows. Upon photo-excitation, excitons are produced in the absorbing medium, diffuse to the interface between the donor polymer and the acceptor fullerene where exciton dissociation can result in free charge carrier generation. Resultant holes in the donor polymer and electrons in the acceptor fullerene are transported to the anode and cathode, respectively. Accordingly, both efficient charge generation as well as efficient charge transport are essential for achieving high performance devices. In this context, the morphology of the $\mathrm{BHJ}$ has received great attention with significant efforts being made to clarify the relationship between film morphology and device performance. ${ }^{1,2}$

Recently, diketopyrrolopyrrole (DPP) based polymers have attracted interest in the OPV community based on beneficial solar light harvesting properties owing to a low bandgap and the ability to tune $\mathrm{BHJ}$ blend morphology through the selection of processing solvents. ${ }^{4-6}$ In this paper, we have investigated $\mathrm{BHJ}$ films based on poly $\{3,6$-dithiophene2-yl-2,5-di(2-octyldodecyl)-pyrrolo[3,4-c]pyrrole-1,4-dione-alt-naphthalene\}(PDPP-TNT) and $[6,6]$-phenyl $C_{71}$-butyric acid methyl ester $\left(P_{71} B M\right)$, Scheme 1. OPV devices utilizing PDPP-TNT and $\mathrm{PC}_{71} \mathrm{BM}$ have shown power conversion efficiencies (PCE) around $5 \% .{ }^{8}$ In 
previous work, we investigated the relationship between device performance, BHJ morphology and the solvent system used for spin coating the active layer. OPV devices prepared from a chloroform: dichlorobenzene (4:1) mixed solvent system showed a higher performance $(\mathrm{PCE}=4.5 \%)$ than those prepared from a chloroform solution $(\mathrm{PCE}=1.9 \%){ }^{8}$ BHJ films prepared from mixed solvents (mix film) consisted of PDPP-TNT "nanofibers", around $15-20 \mathrm{~nm}$ in diameter and $<300 \mathrm{~nm}$ in length, within a surrounding $\mathrm{PC}_{71} \mathrm{BM}$ matrix. In contrast, BHJ films prepared from chloroform (CF film) displayed a well-separated "cobblestone-like" structure where large $\mathrm{PC}_{71} \mathrm{BM}$ aggregates were surrounded by thin "valleys" of PDPP-TNT. Energy filtered TEM was used to distinguish between polymer-rich and fullerene-rich regions within the $\mathrm{BHJ}$ films. The $\mathrm{PC}_{71} \mathrm{BM}$ could be rinsed away from the film with hexane. Fluorescence quenching measurements of films before and after rinsing away the fullerene suggested that PDPP-TNT and $\mathrm{PC}_{71} \mathrm{BM}$ were well segregated in both films. It is noted that such a phase-segregated structure observed in PDPP-TNT:PC ${ }_{71} B M$ is different from that in $\mathrm{P} 3 \mathrm{HT}$ :PCBM films, in which intercalation between $\mathrm{P} 3 \mathrm{HT}$ and PCBM is often observed. ${ }^{9}$ The large difference in $\mathrm{BHJ}$ morphology, still utilizing the same polymer: fullerene components, makes this an interesting system to study charge generation and recombination.

Previously, we investigated charge carrier dynamics in PDPP-TNT:PC ${ }_{71} \mathrm{BM}$ devices using photoinduced charge extraction by linearly increasing voltage (PhotoCELIV). Accordingly, we evaluated charge carrier mobility in a device made from mixed solvents to be $\mu=0.7 \times 10^{-3} \mathrm{~cm}^{2} \mathrm{~V}^{-1} \mathrm{~s}^{-1}$ at $300 \mathrm{~K}^{10}$ Furthermore, from that investigation, we concluded that charge recombination is limited by diffusion of charges, namely Langevin-type recombination. This is in contrast with charge recombination kinetics in $\mathrm{P} 3 \mathrm{HT}$ :PCBM, in which non-Langevin-type recombination is often observed. ${ }^{11,12}$ We also studied exciton dynamics in pristine PDPP-TNT films using time-resolved fluorescence and absorption spectroscopies. $^{13}$

There have been reports in the literature of short singlet exciton lifetime in DPP-based polymers (35 ps) ${ }^{14}$. A shorter singlet exciton lifetime for DPP based polymers compared to $\mathrm{P} 3 \mathrm{HT}(\sim 400 \mathrm{ps})^{15}$ has been attributed to the smaller difference in HOMO-LUMO energy levels of DPP polymers (band-gap from 1.3 to $1.7 \mathrm{eV}$ ) compared to $\mathrm{P} 3 \mathrm{HT}(2.1 \mathrm{eV}) .{ }^{14}$ In contrast, for PDPP-TNT the singlet exciton lifetime was evaluated to be $185 \mathrm{ps},{ }^{13}$ which is comparable to that for $\mathrm{P} 3 \mathrm{HT}$. This indicates that the low band gap nature alone is not a critical factor for singlet exciton lifetime for DPP based polymers, suggesting that a long diffusion length of singlet excitons is feasible. Under high-density excitation 
conditions, the annihilation of excitons was observed and the rate constant was estimated to be $6.0 \times 10^{-9} \mathrm{~cm}^{3} \mathrm{~s}^{-1}$ for PDPP-TNT. ${ }^{13}$ Based on these results, the diffusion length of the singlet exciton was estimated to be about $7 \mathrm{~nm}$. This is comparable with the value obtained in $\mathrm{P} 3 \mathrm{HT}(5-20 \mathrm{~nm})^{16}$.

In this paper, we report the results of transient absorption (TA) and time-resolved microwave conductivity (TRMC) measurements. These were used to further investigate charge generation and recombination kinetics in PDPP-TNT:PC ${ }_{71} B M B H J$ films. Femtosecond TA (fs-TA) was used to evaluate charge generation dynamics in mix and CF $\mathrm{BHJ}$ films. Nanosecond TA (ns-TA) and time-resolved microwave conductivity (TRMC) were used to quantitatively evaluate the recombination of charge carriers. TRMC measurements revealed a long-lived signal that was not observed using ns-TA. The origin of this signal is attributed to the film morphology, microstructure and the resultant electronic properties of spatially isolated charge carriers. Based on this systematic study of charge carrier dynamics, we will discuss factors influencing the performance of PDPP-TNT:PC ${ }_{71}$ BM-based OPV devices.

\section{Experimental}

PDPP-TNT was synthesized in-house as described in previous work. ${ }^{7} \mathrm{PC}_{71} \mathrm{BM}$ was purchased from Sigma-Aldrich and used without further purification. Solutions were prepared by dissolving and stirring PDPP-TNT and $\mathrm{PC}_{71} \mathrm{BM}$ (weight ratios of $1: 2$ ) in chloroform at $60{ }^{\circ} \mathrm{C}$ for several hours. The solution was split into two batches and ortho-dichlorobenzene (DCB) was added to one batch to constitute a ratio of $4: 1$ chloroform : DCB (Mix film). Additional chloroform was added to the other batch so as to maintain the same solute concentration (CF film). Pristine and blend films were prepared on glass substrate by spin-coating technique. Absorption spectra of both blend and pristine films are available in supplementary information in reference 8.

Femtosecond transient absorption measurements were carried out with a Ti: sapphire regenerative amplifier (RegA9000, Coherent) as a light source. The output from the regenerative amplifier ( $800 \mathrm{~nm}$, pulse width of $180 \mathrm{fs}$, and repetition of $10 \mathrm{kHz}$ ) was separated into two beams for the excitation of two optical parametric amplifiers (OPAs) (OPA9400 and OPA9800, Coherent). Pump and probe light pulses were obtained from the two OPAs. For the pump pulse, the output from one OPA at a wavelength of $650 \mathrm{~nm}$ was used. For the probe pulse, $740 \mathrm{~nm}$ light pulse (corresponding to the lowest absorption peak of PDPP-TNT) generated from the other OPA was used to monitor the ground-state 
bleaching signals. The delay time of the probe pulse relative to the pump pulse was controlled by changing the path length of the pump pulse. The time resolution of the system was about 250 fs.

Nanosecond time-resolved TA measurements were carried out with a $\mathrm{Nd}^{3+}$ :YAG laser (Surelite II, HOYA Continuum). The second harmonic (532 nm) of the laser was used for excitation. A xenon lamp (Hamamatsu C7535, $150 \mathrm{~W}$ ) was used as the probe light source. The light transmitted through the sample films was broadly monochromatized (700-750 nm range) with several optical filters and detected with a Si photodiode (DET36A, Thorlabs). The photocurrent from the detector was amplified with an amplifier (DHPVA-100, Femto), and signals were processed with a digital oscilloscope (TDS380, Tektronix) and analyzed with a computer. The DC offset of the photocurrent from the detector was subtracted by using the AC-coupled mode of the amplifier. As a result, very small absorbance changes $(\triangle A$ $<10^{-5}$ ) were detected with 20 ns time resolution. The intensity of the laser pulse was measured with a pyroelectric energy meter (PE25-C, Ophir).

Time-resolved microwave conductivity (TRMC) measurements were carried out with homemade TRMC equipment. ${ }^{17}$ The $\mathrm{Nd}^{3+}$ :YAG laser (Surelite II, HOYA Continuum) was used as the excitation light source. The second harmonic $(532 \mathrm{~nm})$ of the laser was used for excitation. Microwaves were generated by an oscillator based on a Gunn diode (MGS-15B, $200 \mathrm{~mW}$; Nakadai). The oscillator was equipped with a varactor diode, which was used to tune the frequency of the microwaves from 8.5 to $10 \mathrm{GHz}$ (X-band). The microwaves traveled toward the sample cavity through a circulator. Reflected microwaves were detected with a diode (IN23WE, NEC). The photocurrent from the detector was amplified with an amplifier (DHPVA-100, Femto), and signals were processed with a digital oscilloscope (TDS5032, Tektronix) and analyzed with a computer. The time resolution of the system was about 20 ns.

\section{Results and Discussion}

[Femtosecond TA measurements]

fs-TA signals were measured at different excitation light pulse energies in films of pristine PDPP-TNT as well as PDPP-TNT: PC $_{71}$ BM blends prepared from chloroform (CF) and from mixed solvents (Mix). Normalized temporal profiles of the fs-TA signals divided by the excitation light energy absorbed by a sample specimen per pulse, $l_{\text {abs, }}$, are shown in Figure 1. The fraction of absorbed light, $f_{\mathrm{abs}}$, was $0.67,0.29$, and 0.28 for the pristine, the CF and the Mix films, respectively. The fs-TA signal was measured at $740 \mathrm{~nm}$ where PDPP-TNT 
strongly absorbs; bleaching of the ground state absorption was observed. Figure 2 a shows TA signals at just after the excitation pulse, $0.25 \mathrm{ps}$ (when the TA signal is at its maximum), as a function of $I_{\text {abs}}$; the signals were proportional to $I_{\text {abs. }}$. This indicates that no signals faster than time-resolution of our apparatus were included. For the pristine PDPP-TNT films (Fig. 1a), the ground state bleaching signal recovered rapidly with a time constant of 175 ps, which is similar to values obtained previously under relatively strong excitation light intensities. ${ }^{13}$ As we discussed previously, the bleaching recovery profiles are similar to the fluorescence decay profiles, indicating that the ground state is directly repopulated from singlet state decay in the pristine film. We note that there is no contribution due to a long-lived triplet excited state, indicating inefficient intersystem crossing. In contrast with the pristine film, a long-lived component was observed for the blend films as shown in Figs. $1 b$ and 1c. For the blend films, efficient charge generation is expected from the observed incident photon-to-electron conversion efficiency (IPCE) values in devices ${ }^{7}$ and therefore this long-lived component can be associated with the presence of charge carriers. It is noted that the magnitude of the long-lived signal is larger for the Mix films than for the CF films. This is in agreement with the OPV device performance where Mix film devices produce a larger short circuit current density than CF film devices. ${ }^{7}$ Additionally, the decay times of long-lived components can be seen to become shorter with increasing $l_{\text {abs. }}$. This indicates that the long-lived decay profiles are dominated by second order charge recombination; in other words, geminate recombination is not observed in this time range. This is in contrast with the results for other DPP polymer blend films, for which geminate recombination was clearly observed. ${ }^{14}$

Figure $2 \mathrm{~b}$ shows $-\Delta A$ at 2000 ps as a function of $I_{\text {abs. }}$. It can be seen that for $I_{\text {abs }}>$ $0.1 \mu \mathrm{Jcm}^{-2},-\Delta A$ is proportional to $I_{\mathrm{abs}}{ }^{0.5}$ while for $I_{\mathrm{abs}}<0.1 \mu \mathrm{Jcm}^{-2},-\Delta A$ is proportional to $l_{\text {abs }}$ suggesting that nonlinear effects such as exciton annihilation can be effectively ruled out at low $I_{\text {abs. }}$. To discuss the origin of the bleaching signals in detail, temporal profiles under similarly low $I_{\text {abs }}\left(I_{\text {abs }} \sim 0.1 \mu \mathrm{Jcm}^{-2}\right)$ are compared in Fig. 3. The signals at just after the excitation pulse $(0.25 \mathrm{ps})$ are almost the same for the pristine film, Mix film and CF film, suggesting that the number of primary photoproducts is similar. For the pristine film, the signal recovers rapidly and disappears within 1 ns. As we discussed previously, this indicates that the primary photoproduct is the singlet excited state which relaxes back to the ground state through radiative and non-radiative transitions. For the blend films, a similar fast decay component associated with the singlet excited state is observed, followed by a long-lived component, associated with the existence of charge carriers. 
To estimate the contribution of this long-lived component in the blend films, each decay profile was fitted to a single exponential function with an offset value (broken lines in Fig. 3). The exponential term is associated with the fast decay of the singlet excited state, while the offset value is associated with the long-lived charge carriers. The obtained time constants were 175 ps for the pristine film, 124 ps for the CF film, and 92 ps for the Mix film. The offset values, associated with charge carriers, are quite different among the films: 0 for the pristine film, $0.9 \times 10^{-4}$ for the CF film and $1.5 \times 10^{-4}$ for the Mix film. Note that the offset value is correlated with the time constant (fits with shorter time constants have larger offset values). This clearly indicates that the efficiency of charge generation is limited by the quenching rate of singlet excited states, in other words, the majority of singlet excitons undergo charge separation at the interface between PDPP-TNT and $\mathrm{PC}_{71} \mathrm{BM}$ in the blend films. The primary quantum yield of charge generation can be roughly evaluated from the signal intensity of the long-lived species divided by that of the primary species (the ratio of the offset value to the signal value at just after excitation). Accordingly, the yield values are evaluated to be 0.7 for the Mix films and 0.37 for the CF films. This is consistent with our previous study where fluorescence quenching was roughly 0.7 and 0.3 for Mix films and CF films, respectively. ${ }^{8}$ The difference in charge carrier generation efficiency between the CF film and the Mix film is consistent with the difference in the BHJ morphology of the films. For the $\mathrm{CF}$ films, "cobblestone-like" structures are observed where $\mathrm{PC}_{71} \mathrm{BM}$ domains, several hundred nanometers in diameter, are separated by PDPP-TNT, whereas "nanofibers" of PDPP-TNT, around 15-20 nm in diameter and $<300 \mathrm{~nm}$ in length, dispersed throughout a $\mathrm{PC}_{71} \mathrm{BM}$ matrix were observed for the Mix films. ${ }^{8}$ It is clear that smaller sized domains in the Mix film are more favorable for efficient charge generation because the majority of excitons can reach an interface between PDPP-TNT and $\mathrm{PC}_{71} \mathrm{BM}$ within their lifetime. Figure 4 illustrates the difference of the primary processes of charge carrier generation between the CF and Mix films; the efficiency of charge generation is effectively reduced by the relaxation of the exciton to the ground state before reaching the interface.

The quantum yield of charge generation is compared with IPCE measurements at $650 \mathrm{~nm}$ light excitation. IPCE values of 0.6 and 0.2 were found for devices based on Mix film and CF film, respectively. ${ }^{8}$ Although the film thickness of the device $(110 \mathrm{~nm})$ is thicker than that of the samples for fs-TA $(25 \mathrm{~nm})$, similarity between IPCE value and the quantum yield of charge generation suggests that the IPCE value is mainly limited by the quantum yield of charge generation and, in other words, charge collection in the device is highly efficient (perhaps more so for the device employing a Mix film). 


\section{[Evaluation of charge recombination rate constant of the Mix films]}

Figure 5 shows temporal profiles of ns-TA signals observed in the Mix films; these signals were recorded at $700-750 \mathrm{~nm}$, this is near the absorption maximum of the film and is due to PDPP-TNT. Excitation was made by a $532 \mathrm{~nm}$ laser pulse and the fraction of absorbed light $f_{\text {abs }}$ was 0.2 at this wavelength. Similar to the fs-TA measurements (Figs. 1 and 3 ), bleaching of the ground state absorption was observed. As discussed above, the bleaching signal observed here, which recovers in several hundreds of nanoseconds, is mainly associated with charge carriers.

As seen in Fig. 5 , the recovery rate is lower at lower $l_{\text {abs }}$, indicating that second order charge recombination dominates the time profiles, consistent with fs-TA results (Fig. 1). Figure 6 shows that absorbance change $(-\Delta A)$ just after excitation as a function of $l_{\text {abs. }}$. The absorbance change is proportional to $l_{\text {abs }}$ under weak excitation conditions and becomes proportional to $I_{\mathrm{abs}}{ }^{0.5}$ above $I_{\mathrm{abs}}=0.2 \mu \mathrm{Jcm}^{-2}$. Note that very weak excitation laser fluence $F_{\text {laser }}\left(=I_{\text {abs }} / f_{\text {abs }}<1 \mu \mathrm{Jcm}^{-2}\right)$ was needed to observe a linear dependence; highly sensitive TA measurements are essential for studying such efficient recombination processes.

TA techniques have been used to investigate charge recombination in OPV materials. In studies on MDMO-PPV:PC ${ }_{61} \mathrm{BM}^{18}$ and $\mathrm{P} 3 \mathrm{HT}: \mathrm{PC}_{61} \mathrm{BM}^{19}{ }^{19}$ the TA signal dependence on excitation fluence, $F_{\text {laser }}$, showed similar behavior. At high excitation fluence, a fast-decay signal with a nanosecond time constant was observed. A slow decay with a power-law dependence in the millisecond time region became dominant at low $F_{\text {laser. }}$. Interestingly, the slow decay profiles were not sensitive to $F_{\text {laser, }}$ and signal intensities tended to saturate with increasing $F_{\text {laser }}$. Such phenomena have been explained based on a trap-filling model. ${ }^{20,21}$ Similarly, a slow decay in the TA signal was observed for a DPP polymer-based system, however with a different exponent in the power-law decay. ${ }^{14}$ For PDPP-TNT:PC ${ }_{71} B M$, on the contrary, only the fast-decay component can be seen even at low $F_{\text {laser, }}$ as shown in Fig. 5 . This implies that the density of traps in PDPP-TNT:PC ${ }_{71} B M$ films is significantly lower than that of other OPV materials studied by TA previously. This is probably related to the well-segregated structure between PDPP-TNT and $\mathrm{PC}_{71} \mathrm{BM}$ in the PDPP-TNT:PC ${ }_{71} B M$ BHJ films. ${ }^{8}$

Charge recombination kinetics can be expressed using the following rate equation

$$
\frac{\mathrm{d}[N]}{d t}=\frac{\Phi_{\mathrm{CS}}}{\tau_{\mathrm{p}} h v_{e x} d} I_{\mathrm{abs}}-\gamma_{\mathrm{eh}}[N]^{2}
$$

where $[N]$ is the charge density, $\Phi_{\mathrm{CS}}$ is the efficiency of free charge carriers escaped from 
geminate pair, $\tau_{\mathrm{p}}$ is the pulse duration (10 ns), $h$ is the Planck constant, $v_{\mathrm{ex}}$ is the frequency of excitation light, $d$ is the thickness of the films $(60 \mathrm{~nm})$ and $\gamma_{\mathrm{eh}}$ is the rate constant of bulk recombination of charge carriers. As shown in Fig. 6 , the $\Delta \mathrm{A}$ is proportional to $l_{\text {abs }}$ under weak excitation conditions and the signal tends to saturate at $l_{\text {abs }} \geq 0.2 \mu \mathrm{Jcm}^{-2}$. This tendency clearly shows that charge recombination occurs within the time resolution of the TA equipment (20 ns) above $l_{\text {abs }}=0.2 \mu \mathrm{Jcm}^{-2}$. Accordingly, $\gamma_{\mathrm{eh}}$ can be obtained from $\gamma_{\mathrm{eh}}[N]=$ (1 / 20 ns) and $[N]$ can be evaluated as

$$
[N]=\frac{\Phi_{\mathrm{CS}}}{h v_{e x} d} I_{\mathrm{abs}}
$$

Actually, the absolute value of $\Phi_{\mathrm{CS}}$ is not available and therefore the IPCE value $(0.5$ at 532 $\mathrm{nm})^{8}$ observed for a device based on a similarly prepared blend film. It is noted that $\Phi_{\mathrm{CS}}$ values estimated from femtosecond TA measurements (Fig. 3) are similar to those of IPCE. Using the values of $I_{\text {abs }}=0.2 \mu \mathrm{Jcm}^{-2}, \gamma_{\mathrm{eh}}$ can be estimated to be $\gamma_{\mathrm{eh}}=1 \times 10^{-9} \mathrm{~cm}^{3} \mathrm{~s}^{-1}$.

If charge recombination is limited by diffusion (Langevin-type recombination), the $\gamma_{\text {eh }}$ can be expressed as

$$
\gamma_{e h}=e \mu / \varepsilon \varepsilon_{0}
$$

where $e$ is the elementary charge, $\mu$ is the mobility, $\varepsilon$ is the dielectric constant and $\varepsilon_{0}$ is the permittivity in vacuum. Assuming $\varepsilon=3$, which is the typical value for organic materials, $\mu$ can be evaluated to be $\mu=5 \times 10^{-4} \mathrm{~cm}^{2} V^{-1} \mathrm{~s}^{-1}$ from the estimated recombination rate constant $\left(\gamma_{\mathrm{eh}}\right.$ $=1 \times 10^{-9} \mathrm{~cm}^{3} \mathrm{~s}^{-1}$ ). This mobility value is similar to that obtained from PhotoCELIV measurements in pDPP-TNT:PC ${ }_{71} \mathrm{BM}\left(\mu=7 \times 10^{-4} \mathrm{~cm}^{2} \mathrm{~V}^{-1} \mathrm{~s}^{-1}\right.$. at $\left.300 \mathrm{~K}\right){ }^{10}$ This indicates that the charge recombination in PDPP-TNT:PC ${ }_{71} \mathrm{BM}$ is limited by diffusion of charges (Langevin-type recombination). This is consistent with the conclusion obtained from the saturation of photoinduced current at high laser intensities in PhotoCELIV measurements. ${ }^{10}$ This is in contrast with charge recombination kinetics in $\mathrm{P} 3 \mathrm{HT}: \mathrm{PC}_{61} \mathrm{BM}$, in which non-Langevin-type recombination is often observed. ${ }^{11,12}$ Recently, Clarke et al. have studied the effect of polymer structure on non-Langevin-type recombination kinetics. ${ }^{22}$ Accordingly, they pointed out that amorphous mixed phase between crystalline polymer and crystalline fullerene plays an important role for non-Langevin-type recombination. As studied previously, ${ }^{8}$ PDPP-TNT and PC $_{71} \mathrm{BM}$ is well segregated in PDPP-TNT: PC71BM blend films, in other words, mixed intercalated phase between PDPP-TNT and $\mathrm{PC}_{71} \mathrm{BM}$ is a minor part. This would be a possible reason for observing Langevin-type recombination in PDPP-TNT:PC ${ }_{71} B M$ blend films. 
For pristine PDPP-TNT films, the hole mobility was estimated to be $\mu=0.98$ $\mathrm{cm}^{2} \mathrm{~V}^{-1} \mathrm{~s}^{-1}$ from field effect transistor (FET) characteristics, ${ }^{7}$ which is three orders of magnitude larger than those obtained from PhotoCELIV $\left(\mu=7 \times 10^{-4} \mathrm{~cm}^{2} \mathrm{~V}^{-1} \mathrm{~s}^{-1}\right)$ and TA $(\mu=$ $5 \times 10^{-4} \mathrm{~cm}^{2} \mathrm{~V}^{-1} \mathrm{~s}^{-1}$ ) measurements for PDPP-TNT:PC ${ }_{71}$ BM blend films. This discrepancy can be attributed to a difference in experimental conditions. FET characteristics are examined under high charge density conditions, which is significantly different from other techniques such as PhotoCELIV. In fact, it has been clearly shown that charge mobility increases dramatically with increasing charge density. ${ }^{23}$ It was reported that mobility ( $\mu=2 \times 10^{-3}$ $\mathrm{cm}^{2} \mathrm{~V}^{-1} \mathrm{~s}^{-1}$ ) obtained by time-of-flight (TOF) and space charge limited current (SCLC) measurements examined under low charge density condition was much lower than mobility $\left(\mu>10 \mathrm{~cm}^{2} V^{-1} \mathrm{~s}^{-1}\right)$ obtained by FET characterization for pristine DPP based polymer (PDPPDTSE) films. ${ }^{24}$

\section{[Comparison of TA signal with TRMC signal]}

TRMC is a type of TA spectroscopy using microwaves as a probe to measure the conductivity $(\sigma)$ of mobile charges generated by light excitation. ${ }^{25}$ The absorption of microwave power $(-\Delta P / P)$ can be expressed as

$$
\left(\frac{-\Delta P}{P}\right)=K \sigma=K^{\prime} \mu N_{\mathrm{C}}
$$

where $K$ and $K^{\prime}$ are constants, $\mu$ is the mobility of the charge carriers, and $N_{\mathrm{C}}$ is the number of charge carriers present. Note that TA signals include only information about the number of charge carriers, whereas both the number and mobility of charge carriers contribute to TRMC signals. Accordingly, information about mobility of charge carriers can be extracted by making a comparison between TA and TRMC measurements. Figure 7 shows the temporal profiles of TA and TRMC signals under strong $\left(I_{\text {abs }}=0.8 \mu \mathrm{Jcm}^{-2}\right)$ and weak $\left(I_{\text {abs }}=\right.$ $0.06 \mu \mathrm{Jcm}^{-2}$ ) excitation conditions in the Mix films. It is obvious that TRMC signals include both fast and slow decay components, whereas only a fast decay component can be seen in the TA signals.

As shown in Fig. 7, the fast components of the TA and TRMC profiles seem to be similar to each other and the slow components for TRMC show almost no change with time. Thus, the fast components of TA and TRMC profiles can be directly compared when a constant value (shaded area) is added to the TA signals as shown at the bottom of Fig. 7. It is clear that the fast component of TA signals shows similar decay to that of TRMC signals. 
This indicates that the majority of charge carriers undergo recombination in this time range. This suggests that the slow component observed in the TRMC signal is due to a minor amount of long-lived charge carriers. Consequently, these charges must have a high mobility due to the relative strength of the long-lived TRMC signal. We further discuss the influence of $\mathrm{BHJ}$ morphology and thin film microstructure on charge carrier dynamics in conjunction with the presence of long-lived, high mobility charge carriers.

Although the absolute value of conductivity is difficult to evaluate in our TRMC apparatus because of a lack of reliable standard samples, it is interesting to discuss our results in comparison to quantitative TRMC studies of $\mathrm{P} 3 \mathrm{HT}: \mathrm{PC}_{61} \mathrm{BM}$ films ${ }^{26}$ The observed conductivity $\sigma\left(=\mu \Phi_{\mathrm{CS}}\right)$ of charge carriers increased dramatically after thermal annealing of $\mathrm{P} 3 \mathrm{HT}: \mathrm{PC}_{61} \mathrm{BM}$ films. This was explained by the growth of $\mathrm{PC}_{61} \mathrm{BM}$ crystallites in the $\mathrm{P} 3 \mathrm{HT}$ polymer matrix. After adequate annealing, the TRMC measured mobility reached a value of $1 \times 10^{-1} \mathrm{~cm}^{2} \mathrm{~V}^{-1} \mathrm{~s}^{-1}$, which is similar to that of polycrystalline $\mathrm{PC}_{61} \mathrm{BM}$ films obtained by TRMC measurements $\left(4 \times 10^{-2} \mathrm{~cm}^{2} \mathrm{~V}^{-1} \mathrm{~s}^{-1}\right.$ and $\left.3 \times 10^{-1} \mathrm{~cm}^{2} \mathrm{~V}^{-1} \mathrm{~s}^{-1}\right){ }^{27}$ This indicated that the TRMC signal for $\mathrm{P} 3 \mathrm{HT}: \mathrm{PC}_{61} \mathrm{BM}$ films was dominated by the mobility of electrons in crystalline $\mathrm{PC}_{61} \mathrm{BM}$. It is reasonable to expect the electron mobility in crystalline $\mathrm{PC}_{61} \mathrm{BM}$ to be larger than the hole mobility in the polymer domains at room temperature. It should be noted that the electron mobility of $C_{60}$ single crystals has been evaluated to be $1.1 \pm 0.1 \mathrm{~cm}^{2} V^{-1} \mathrm{~s}^{-1}$ by the time-of-flight (TOF) method, while mobility values of $\mathrm{PC}_{61} \mathrm{BM}$ and $\mathrm{PC}_{71} \mathrm{BM}$ single crystals are not available. ${ }^{28}$

As discussed above, the $\mathrm{PC}_{61} \mathrm{BM}$ crystallite growth that occurs through thermal annealing of $\mathrm{P} 3 \mathrm{HT}: \mathrm{PC}_{61} \mathrm{BM}$ films has been associated with increased polymer fullerene phase segregation. We previously reported that PDPP-TNT and $\mathrm{PC}_{71} \mathrm{BM}$ are well segregated in PDPP-TNT:PC $C_{71} B M$ films due to the fact that $\mathrm{PC}_{71} \mathrm{BM}$ could be rinsed away from the film by hexane without removing any polymer. ${ }^{8}$ This segregation is beneficial for the transport of holes in the polymer and electrons in the fullerene. Accordingly, we attribute the TRMC signal seen in PDPP-TNT:PC ${ }_{71} B M$ to electrons in $\mathrm{PC}_{71} \mathrm{BM}$, similar to the TRMC signal in annealed $\mathrm{P} 3 \mathrm{HT}: \mathrm{PC}_{61} \mathrm{BM}$ films.

TRMC measured mobility in polycrystalline $\mathrm{PC}_{61} \mathrm{BM}$ films $\left(4 \times 10^{-2} \mathrm{~cm}^{2} \mathrm{~V}^{-1} \mathrm{~s}^{-1}\right.$ and 3 $\left.\times 10^{-1} \mathrm{~cm}^{2} \mathrm{~V}^{-1} \mathrm{~s}^{-1}\right)^{27}$ is much larger than that of PDPP-TNT:PC ${ }_{71} B M$ blend films obtained from PhotoCELIV $\left(\mu=7 \times 10^{-4} \mathrm{~cm}^{2} \mathrm{~V}^{-1} \mathrm{~s}^{-1}\right)$ and TA $\left(\mu=5 \times 10^{-4} \mathrm{~cm}^{2} \mathrm{~V}^{-1} \mathrm{~s}^{-1}\right)$ measurements. This difference can be attributed to the type of charge carrier motion being probed. ${ }^{9}$ Since TRMC is an AC conductivity measurement, the signal intensity reflects back-and-forth drift motion 
of charge carriers within $100 \mathrm{ps}$, induced by the microwave electric field (9 GHz). Accordingly, TRMC mobility is strongly affected by microstructure of the sample. In this context, the relatively low mobility in PDPP-TNT:PC ${ }_{71} B M$ obtained from PhotoCELIV and TA in the present study suggests that the motion of the majority of electrons in $\mathrm{PC}_{71} \mathrm{BM}$ domain is significantly influenced by trapping. Grain boundaries in $\mathrm{PC}_{71} \mathrm{BM}$ domains are most probably responsible for such charge carrier trapping.

Next, the difference between the ns-TA and TRMC signal transients for PDPP-TNT:PC ${ }_{71} B M$ Mix films (Fig. 7) will be addressed. As discussed above, the TRMC signal in the present study is composed of two components, fast and slow decay signals. The fast-decay component reflects charge recombination of the majority of charge carriers as evidenced by the fact that the decay profiles are similar to the recovery profiles of the ns-TA signals. This indicates that the majority of charge carriers, for which the motion is dictated by trapping at grain boundaries between $\mathrm{PC}_{71} \mathrm{BM}$ crystallites, undergo fast recombination. As discussed above, the long-lived component in the TRMC transients reflects the motion of a minor amount of charge carriers. A large TRMC signal from such a minor amount of charge carriers suggests that these carriers possess a high mobility. High mobility is expected for electrons in $\mathrm{PC}_{71} \mathrm{BM}$ crystallites free of grain boundaries. Accordingly, electrons in single crystal domains isolated from other crystallites are most probably responsible for the long-lived signal in TRMC transients.

For P3HT:PC ${ }_{61} \mathrm{BM}$, a comparative study between ns-TA and TRMC measurements was carried out. ${ }^{29}$ Accordingly, the decay of ns-TA profiles was very similar to that of TRMC in the time range from $100 \mathrm{~ns}$ to $100 \mu \mathrm{s}$. This means that the same charged species were detected by both ns-TA and TRMC for P3HT:PC ${ }_{61} \mathrm{BM}$, which is in contrast with what was observed in the present study for PDPP-TNT:PC ${ }_{71} B M$. This discrepancy could be attributed to differences in charge trapping properties. Namely, for $\mathrm{P} 3 \mathrm{HT}: \mathrm{PC}_{61} \mathrm{BM}$, a majority of charge carriers experience trapping / de-trapping and thus both long-lived ns-TA and TRMC signals are observed. On the contrary, for PDPP-TNT:PC ${ }_{71} \mathrm{BM}$, a majority of charge carriers undergo recombination as observed in ns-TA measurements (Fig. 5). As discussed above, a minor amount of charge carriers, having high mobility, contributes to the long-lived signal of TRMC. This suggests that such charge carriers are located in isolated single crystal domains of $\mathrm{PC}_{71} \mathrm{BM}$, and the number of these carriers is too small to be detected by ns-TA measurements.

In order to discuss the slow components of the TRMC signal in more detail, TRMC 
measurements on the micro-second time scale were carried out under different excitation intensities (Fig. 8). As shown in Fig. 8, the decay profiles are not sensitive to $l_{\text {abs. }}$ This behavior is similar to that observed in TA measurements for $\mathrm{P} 3 \mathrm{HT}: \mathrm{PC}_{61} \mathrm{BM}$ films. ${ }^{19}$ The decay profiles cannot be fit to simple second-order kinetics. The profiles can be fit to a power-law dependence $\left(t^{-\beta}\right)$ using the value $\beta=0.3$. Such a power-law dependence has been often observed in the recombination kinetics of polymer OPV materials measured using TA. ${ }^{18,19}$ Such a power-law dependence has been associated with charge carrier recombination limited by thermally activated de-trapping processes..$^{20,21}$

The characteristics of the decay profiles measured with ns-TA and TRMC can be related to the structure of the $\mathrm{BHJ}$ film. As shown in our previous studies, PDPP-TNT forms a network of nanofibers within a $\mathrm{PC}_{71} \mathrm{BM}$ matrix in Mix films. From the fact that efficient charge collection occurs in Mix films, these nanofibers overlap and form a well-connected percolation path for charge transport. (Figure 9). As mentioned above, PDPP-TNT is well segregated from $\mathrm{PC}_{71} \mathrm{BM}$ in the Mix films; $\mathrm{PC}_{71} \mathrm{BM}$ forms nano-sized crystals which also overlap and provide a charge percolation pathway. Grain boundaries exist between $\mathrm{PC}_{71} \mathrm{BM}$ crystallites within the transport network. Such a film structure can be associated with the majority of charges which undergo recombination which is described by the fast-decay component of TRMC profiles. We propose that a minor amount of highly crystalline $\mathrm{PC}_{71} \mathrm{BM}$ is isolated from the conducting, charge percolation network of $\mathrm{PC}_{71} \mathrm{BM}$. The long-lived TRMC signal can be associated with a minority of electrons that are located in these isolated regions. Electrons in these isolated regions have access to a limited donor-acceptor interfacial area in contrast to electrons in the well-connected percolation network which can travel longer distances and sample a large donor-acceptor interfacial area throughout the film. Thus, the recombination rate of charges in the conducting network is determined by the mobility of electrons while the recombination rate of electrons in isolated regions is limited by the mobility of holes. The power-law dependence in Fig. 8 is in agreement with hole transport dominated by trapping. ${ }^{18-21}$ Accordingly, the isolated electrons may give a strong, long-lived TRMC signal because of high AC-drift mobility and slow recombination.

\section{Conclusion}

Generation and recombination dynamics of charge carriers in PDPP-TNT:PC ${ }_{71} B M$ were studied by means of TA and TRMC. From fs-TA profiles, we conclude that the efficiency of charge generation is not dominated by geminate recombination but limited by exciton diffusion in the films. From ns-TA measurements, the charge recombination rate constant $\gamma_{\mathrm{eh}}$ was estimated to be $\gamma_{\mathrm{eh}}=1 \times 10^{-9} \mathrm{~cm}^{3} \mathrm{~s}^{-1}$, which is similar to that obtained by PhotoCELIV 
previously. From the difference between TA and TRMC transients, we conclude that majority of charge carriers undergo fast recombination owing to their location in a well-connected conduction network. A minor amount of electrons are located in isolated crystalline domains of $\mathrm{PC}_{71} \mathrm{BM}$, free form other crystallites and grain boundaries, and contribute to a long-lived TRMC signal. The charge carrier dynamics of PDPP-TNT:PC ${ }_{71} B M$ films are significantly different from those of $\mathrm{P} 3 \mathrm{HT}: \mathrm{PC}_{61} \mathrm{BM}$ films. This is attributed to a difference in charge trap properties.

\section{Acknowledgements}

This work was funded by the A*STAR-JST Strategic International Cooperative Program (SICP, Project No. 1021630071).

\section{References}

[1] Kippelen, B.; Brédas, J.-L. Organic Photovoltaics. Energy Environ. Sci. 2009, 2, 251-261.

[2] Clarke, T. M.; Durrant, J. R. Charge Photogeneration in Organic Solar Cells. Chem. Rev., 2010, 110. 6736-6767.

[3] Green, M. A.; Emery, K.; Hishikawa, Y.; Warta, W.; Dunlop, E. D. Solar Cell Efficiency Tables (Version 45). Prog. Photovoltaics, 2015, 23, 805-812.

[4] Li, Y. N.; Sonar, P.; Murphy, L.; Hong, W. High Mobility Diketopyrrolopyrrole (DPP)-based Organic Semiconductor Materials for Organic Thin Film Transistors and Photovoltaics. Energy Environ. Sci. 2013, 6, 1684-1710.

[5] Liu, C.; Wang, K.; Gong, X.; Heeger, A. J. Low Bandgap Semiconducting Polymers for Polymeric Photovoltaics. Chem. Soc. Rev. 2016, DOI: 10.1039/C5CS00650C.

[6] Li, W.; Hendriks, K H.; Wienk, M. M.; Janssen, R. A. J. Diketopyrrolopyrrole Polymers for Organic Solar Cells. Acc. Chem. Res., 2016, 49, 78-85.

[7] Sonar, P.; Singh, S. P.; Li, Y.; Ooi, Z.-E.; Ha, T.; Wong, I.; Soh, M. S.; Dodabalapur, A. High Mobility Organic Thin Film Transistor and Efficient Photovoltaic Devices using Versatile Donor-acceptor Polymer Semiconductor by Molecular Design. Energy Environ. Sci. 2011, 4, 2288-2296.

[8] Williams, E. L.; Gorelik, S.; Phang, I. Y.; Bosman, M.; Vijila, C.; Subramanian, G. S.; Sonar, P.; Hobley, J.; Singh, S. P., Matsuzaki, H.; Furube, A.; Katoh R. Nanoscale Phase Domain Structure and Associated Device Performance of Organic Solar Cells Based on a Diketopyrrolopyrrole Polymer. RSC Advances, 2013, 3. 20113-20124.

[9] Savenije, T. J.; Ferguson, A. J. ; Kopidakis, N. ; Rumbles, G. Revealing the Dynamics of Charge Carriers in Polymer: Fullerene Blends using Photoinduced Time-resolved Microwave Conductivity. J. Phys. Chem. C, 2013, 117, 24085-24103 and references 
therein.

[10] Vijila, C.; Singh, S. P.; Williams, E. L.; Sonar, P.; Pivrikas, A,; Philippa, B.; White, R.; Kumar, E. N.; Subramanian, G. S.; Gorelik, S.; Hobley, J.; Furube, A.; Matsuzaki, H.; Katoh R. Relation Between Charge Carrier Mobility and Lifetime in Organic Photovoltaics. J. Appl. Phys. 2013, 114, 184503.

[11] Pivrikas, A.; Juška, G.; Mozer, A. J.; Scharber, M.; Arlauskas, K.; Sariciftci, N. S.; Stubb, H.; Österbacka, R. Bimolecular Recombination Coefficient as a Sensitive Testing Parameter for Low-mobility Solar-cell Materials. Phys. Rev. Lett. 2005, 94, 176806-176806.

[12] Hamilton, R.; Shuttle, C. G.; O'Regan, B.; Hammant, T. C.; Nelson, J.; Durrant, J. R. Recombination in Annealed and Nonannealed Polythiophene/fullerene Solar Cells: Transient Photovoltage Studies Versus Numerical Modeling. J. Phys. Chem. Lett., 2010, 1, 1432-1436.

[13] Matsuzaki, H.; Furube, A.; Katoh R.; Singh, S. P.; Sonar, P.; Williams, E. L.; Vijila, C.; Subramanian, G. S.; Gorelik, S.; Hobley, J. Excited-state Dynamics in Diketopyrrolopyrrole-based Copolymer for Organic Photovoltaics Investigated by Transient Optical Spectroscopy. Jpn. J. Appl. Phys. 2014, 53, 01AB11.

[14] Albert-Seifried, S.; Ko, D.-H.; Hüttner, S.; Kanimozhi, C.; Patilc, S.; Friend, R. H. Efficiency Limitations in a Low Band-gap Diketopyrrolopyrrole-based Polymer Solar Cell. Phys. Chem. Chem. Phys. 2014, 16, 6743-6752.

[15] Cook, S.; Furube, A.; Katoh, R. Analysis of the Excited States of Regioregular Polythiophene P3HT. Energy Environ. Sci. 2008, 1, 294-299 and reference therein.

[16] Tamai, Y.; Ohkita, H.; Benten, H.; Ito, S. Exciton Diffusion in Conjugated Polymers: From Fundamental Understanding to Improvement in Photovoltaic Conversion Efficiency. J. Phys. Chem. Lett. 2015, 6, 3417-3428.

[17] Katoh, R.; Kasuya, M.; Furube, A.; Fuke, N.; Koide, N.; Han. L. Quantitative Study of Solvent Effects on Electron Injection Efficiency for Black-dye-sensitized Nanocrystalline $\mathrm{TiO}_{2}$ Films. Sol. Energy Mater. Sol. Cells 2009, 93, 698-703.

[18] Nogueira, A. F.; Montanari, I.; Nelson, J.; Durrant, J. R.; Winder, C. ; Sariciftci, N. S.; Brabec, C. Charge Recombination in Conjugated Polymer/fullerene Blended Films Studied by Transient Absorption Spectroscopy. J. Phys. Chem. B 2003, 107, 1567-1573.

[19] Clarke, T. M.; Jamieson, F. C.; Durrant, J. R. Transient Absorption Studies of Bimolecular Recombination Dynamics in Polythiophene/fullerene Blend Films. J. Phys. Chem. B 2009, 113, 20934-20941.

[20] Nelson, J. Diffusion-limited Recombination in Polymer-fullerene Blends and Its Influence on Photocurrent Collection. Phys. Rev. B 2003, 67, 155209. 
[21] Tachiya, M.; Seki, K. Theory of Bulk Electron-hole Recombination in a Medium with Energetic Disorder. Phys. Rev. B 2010, 82, 085201.

[22] Clarke, T. M.; Lungenschmied, C.; Peet, J.; Drolet, N.; Mozer, A. J. Tuning Non-Langevin Recombination in an Organic Photovoltaic Blend Using a Processing Additive. The Journal of Physical Chemistry C, 2015, 119, 7016-7021.

[23] Shuttle, C. G.; Hamilton, R.; Nelson, J.; O'Regan, B. C.; Durrant, J. R. Measurement of Charge - Density Dependence of Carrier Mobility in an Organic Semiconductor Blend. Advanced Functional Materials, 2010, 20, 698-702.

[24] Cheon, K. H.; Cho, J.; Lim, B. T.; Yun, H. J.; Kwon, S. K.; Kim, Y. H.; Chung, D. S. Analysis of Charge Transport in High-mobility Diketopyrrolopyrole Polymers by Space Charge Limited Current and Time of Flight Methods. RSC Advances, 2014, 4, 35344-35347.

[25] Kroeze, J. E.; Savenije, T. J.; Warman, J. M. Electrodeless Determination of the Trap Density, Decay Kinetics, and Charge Separation Efficiency of Dye-sensitized Nanocrystalline $\mathrm{TiO}_{2}$. J. Am. Chem. Soc. 2004, 126, 7608-7618.

[26] Grzegorczyk, W. J.; Savenije, T. J.; Dykstra, T. E.; Piris, J.; Schins, J. M.; Siebbeles, L. D. Temperature-independent Charge Carrier Photogeneration in P3HT- PCBM Blends with Different Morphology. J. Phys. Chem. C 2010, 114, 5182-5186.

[27] de Haas, M. P.; Warman, J. M.; Anthopoulos, T. D.; de Leeuw, D. M. The Mobility and Decay Kinetics of Charge Carriers in Pulse-ionized Microcrystalline PCBM Powder. Adv. Funct. Mater. 2006, 16, 2274-2280.

[28] Frankevich, E.; Maruyama, Y.; Ogata, H. Mobility of Charge Carriers in Vapor-phase Grown $\mathrm{C}_{60}$ Single Crystal. Chem. Phys. Lett. 1993, 214, 39-44.

[29] Savenije, T. J.; Murthy, D. H. K.; Gunz, M.; Gorenflot, J.; Siebbeles, L. D.; Dyakonov, V.; Deibel, C. Absence of Postnanosecond Charge Carrier Relaxation in Poly(3-hexylthiophene)/fullerene Blends. J. Phys. Chem. Lett. 2011, 2, 1368-1371. 
Figures

Scheme 1 Molecular structure of poly\{3,6-dithiophene- 2-yl-2,5-di(2-octyldodecyl)pyrrolo[3,4-c]pyrrole-1,4-dione-alt-naphthalene\} (PDPP-TNT) 
Fig. 1 Time profiles of transient absorption normalized by the excitation light energy absorbed by a sample specimen per pulse $l_{\text {abs }}$ in (a) pristine PDPP-TNT film, (b) CF film, and (c) Mix film at various excitation fluences. 
Fig. $2 l_{\text {abs }}$ dependence of transient absorption signal at just after excitation pulse (0.25 ps) (a) and at 2000 ps (b). 


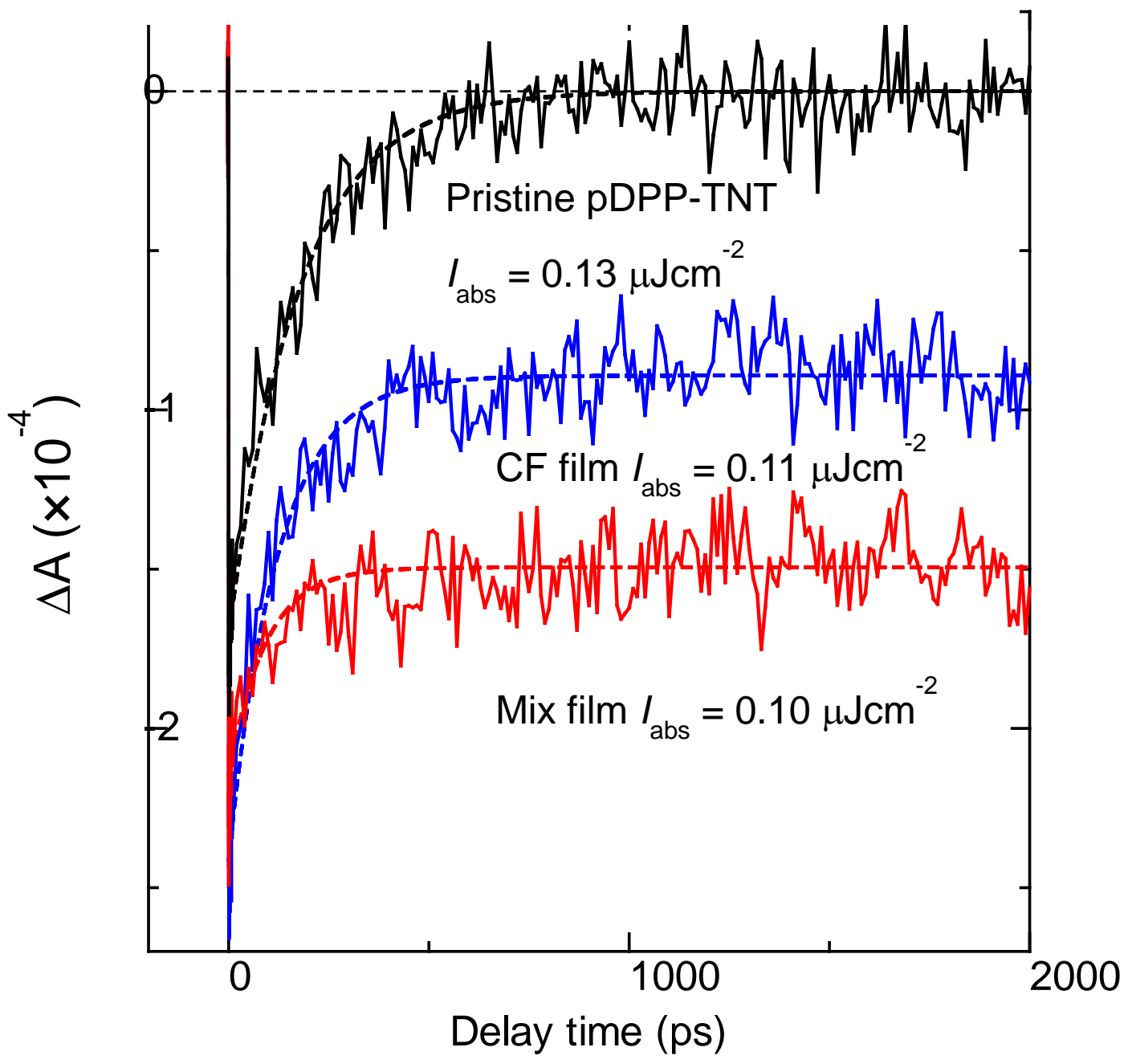

Fig. 3 Time profiles of transient absorption in pristine PDPP-TNT film, CE film, and Mix film under weak ( $\left(I_{\mathrm{abs}} \sim 0.1 \mu \mathrm{Jcm}^{-2}\right)$ excitation conditions (solid lines). The profiles are fitted by single exponential function with an offset value (broken lines). 
Fig. 4 Possible charge generation model for the CF and Mix films of PDPP-TNT:PC ${ }_{71} B M$. The CF film has larger polymer and fullerene domains; the Mix film contains bundles of polymer nanofibers and smaller domains which allow for more efficient charge generation because the majority of excitons can reach an interface between PDPP-TNT and PC ${ }_{71} B M$ within their lifetime. 
Fig. 5 Temporal profiles of TA signals in nano-second time region excited at $l_{\text {abs }}=0.8$ $\mu \mathrm{Jcm}^{-2}$ and $I_{\text {abs }}=0.06 \mu \mathrm{Jcm}^{-2}$ for the Mix films. 
Fig. 6 Absorbance change $(-\Delta \mathrm{A})$ at just after excitation as a function of $l_{\text {abs }}$ for the Mix films. 

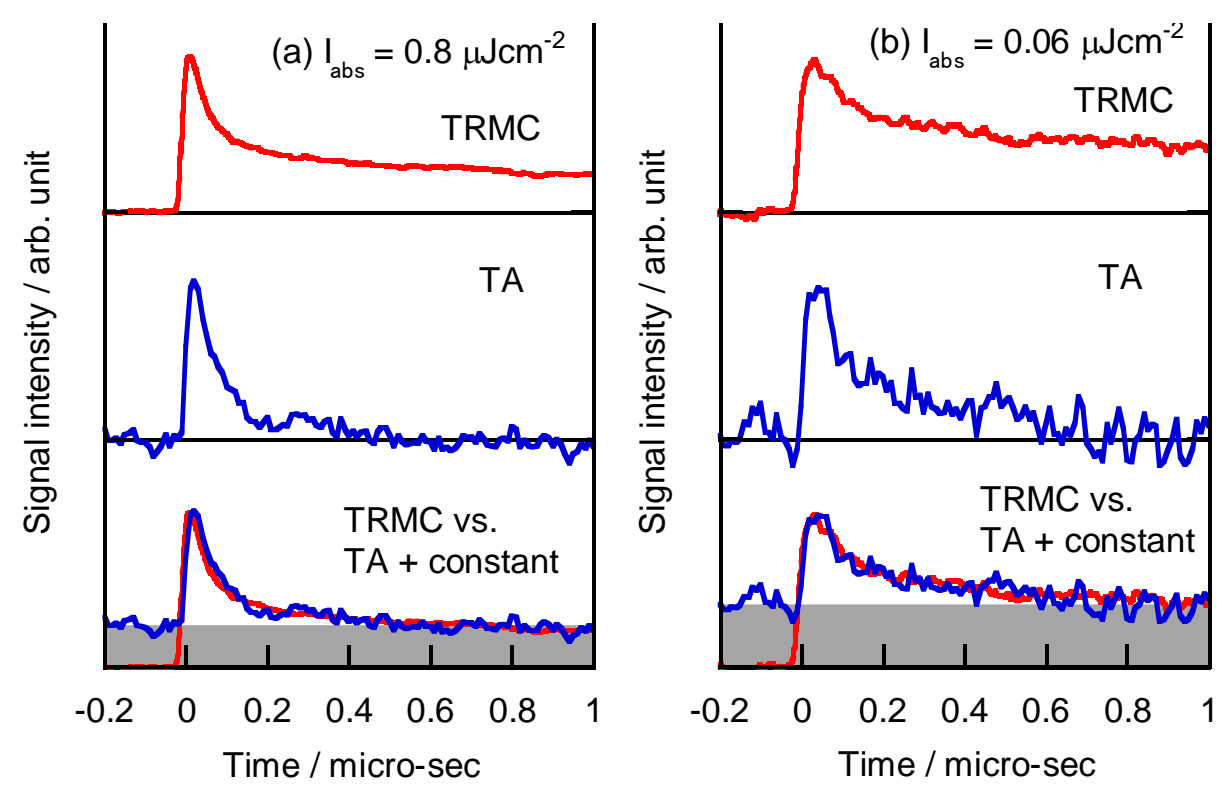

Fig.

7 Comparison of temporal profiles between TA and TRMC signals at strong $\left(l_{\mathrm{abs}}=0.8\right.$ $\left.\mu \mathrm{Jcm}^{-2}\right)$ and weak $\left(I_{\mathrm{abs}}=0.06 \mu \mathrm{Jcm}^{-2}\right)$ excitation conditions for the Mix films. 
Fig. 8 The decay profiles of TRMC in wide time range for the Mix films. 
Fig. 9 Possible structural model for the Mix films of PDPP-TNT:PC ${ }_{71} B M$. Polycrystalline $\mathrm{PC}_{71} \mathrm{BM}$ domains exist amongst a network of PDPP-TNT nanofibers. Grain boundaries may act as electron traps. Isolated regions of $\mathrm{PC}_{71} \mathrm{BM}$ surrounded by PDPP-TNT may allow for long-lived electrons with a high mobility. 


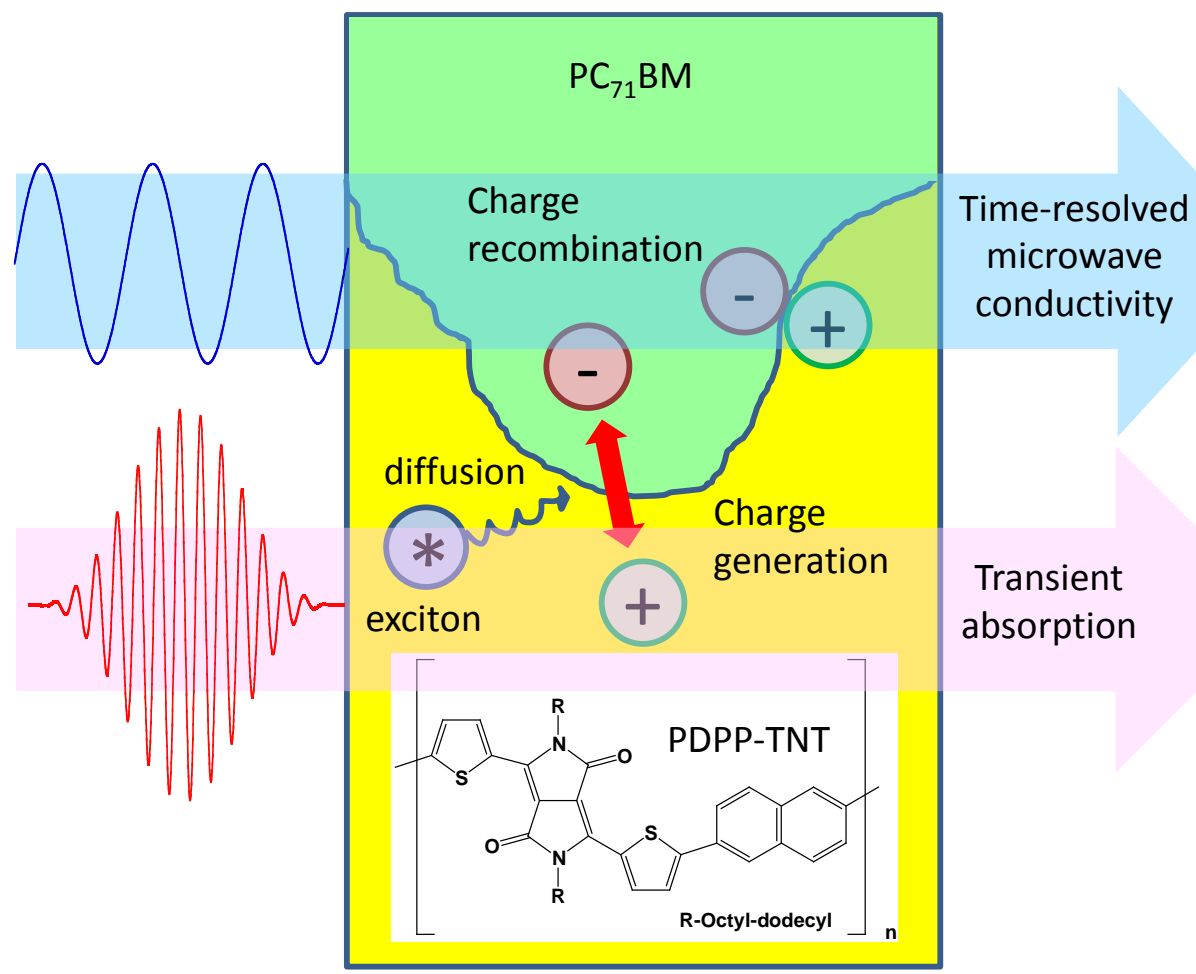

Table of Contents Graphic:

Charge generation and recombination dynamics in organic photovoltaic bulk heterojunction films comprising the donor polymer, PDPP-TNT blended with the fullerene acceptor, $\mathrm{PC}_{71} \mathrm{BM}$ have been studied by means of transient absorption spectroscopy and time-resolved microwave conductivity. 